\title{
The therapeutic behavior of the dentist in patients with atherosclerosis - theoretical and practical aspects
}

\author{
Ioana Voinescu' ${ }^{1}$, Dumitru Ferechide ${ }^{1}$, Viorel Perieanu', Maria Glencora Costache ${ }^{1}$, \\ Octavian Dumitru Zara², Madalina Violeta Perieanu', Claudia-Camelia Burcea', \\ Madalina Malita', Irina Adriana Beuran', Liliana Burlibasa ${ }^{3}$, Narcis Marcov'1, \\ Gabriela lorgulescu', Magdalena Natalia Dina', Mihai Burlibasa', Luminita Oancea', \\ Ileana Ionescu ${ }^{1}$, Elena-Cristina Marcov' ${ }^{1}$ \\ 1"Carol Davila" University of Medicine and Pharmacy, Bucharest, Romania \\ 2"Sf. Ioan" Emergency Clinical Hospital, Bucharest, Romania \\ ${ }^{3}$ Faculty of Biology, University of Bucharest, Romania
}

\begin{abstract}
Introduction. Atherosclerosis represents the process of stiffening of the arterial wall and includes arterial changes secondary to atherosclerosis, as well as physiological changes, reducing vascular elasticity in the elderly. However, the importance of knowing the pathology of atherosclerosis is mandatory among dentists, given the high frequency of patients with atherosclerotic lesions.

Purpose. Through this study we tried to familiarize dentists on the therapeutic behavior towards patients with atherosclerosis but also the risks arising from it.

Material and method. 87 practitioners from Romania helped to carry out this study between September and November 2018.

Results and discussions. A clear picture emerged regarding the changes and manifestations in patients with atherosclerosis by dental practitioners as well as the attitude to be adopted in the case of treatments for such patients.

Conclusions. Knowing the pathology of patients at cardiovascular risk as well as the therapy to be applied for them, is a must for dentists given the growing number of patients and the pathology with different stages of evolution.
\end{abstract}

Keywords: atherosclerosis, dental treatment, periodontitis

\section{INTRODUCTION}

One of the most prevalent conditions affecting human health is atherosclerosis, a systemic evolutionary disease that mainly affects the blood vessels of large and/or medium size (for example, the aorta and its branches etc.). Atherosclerosis is characterized by thickening of the vascular wall and narrowing of the arterial lumen, with ischemic disorders in the affected territory. In general, this con- dition is common after the age of 50, especially in men, due to the coexistence of risk factors, such as alcohol, smoking, or stress, women being protected by estrogen until menopause (1-5).

Atheromatosis of the cervical and cerebral arterial system is localized in humans especially in the proximal region of the cervical and cerebral arteries and, as we move to the cortical vessels, this process is diminished so that both at the level of the cerebral, cervical and facial vessels, but also at the 
level of vessels from the structures of the dento-maxillary system, these processes are minimal. Specifically, these changes can be associated with a number of risk factors, such as: high blood pressure, diabetes, obesity, vascular syphilis, various toxic factors, dyslipidemia, smoking, alcohol consumption, various genetic factors etc. (1-6).

\section{GENERAL DATA}

Atherosclerosis, an evolutionary systemic disease that is associated with other cardiovascular diseases, manifests itself in the oral cavity through osteoporosis processes, to which are added both the resorption of alveolar processes and changes of the temporomandibular joint (1-6). Thus, the epithelium of the oral mucosa is thinned, keratinized, the salivary glands are atrophied and, consequently, the salivary secretion is diminished and more viscous (1-6). Thus, decreased salivary secretion is an important aggravation factor for carious pathology, along with inadequate oral hygiene. In these cases, the mobility of the teeth has a slow evolution, the dental pulp changes its structure due to a very deficient irrigation of its connective tissue, thus reducing its resistance to possible aggression that may occur later. Regarding tooth enamel, it undergoes accentuated wear processes that reduce its thickness, making it quite vulnerable to the aggressions of microbial agents (1-6).

However, periodontal disease is a chronic disease, considered to be a minor, isolated microbial infection, unrelated to stroke, but periodontal disease with damage of alveolar bone involves a considerable bone surface, which comes in contact with considerable areas of soft tissue, through which a lot of bacteria and components of local inflammatory reactions, can enter the blood system and affect the walls of blood vessels (1-6). Nowadays, it is accepted by almost all dental professionals that chronic marginal periodontitis play an important role in the etiology and subsequently in the evolution of cardiac and cardiovascular diseases (1-6).

The presence of a patient with cardiovascular risk after atherosclerosis for a dental treatment requires a very thorough internal medicine consultation before dental treatment (1-6). And, it is absolutely normal to do this, given the scale of a complex dentistry treatment for such patients (1-6). Practically, it is advisable to prevent certain incidents in this type of patients, when they present in the dental office. Next, we will briefly present, some aspects that a dentist should consider when a patients with cardiovascular risk after atherosclerosis came in the dental office.

\section{PURPOSE}

Starting from the aforementioned and before presenting the objectives of this study, the dentist must consider more coordinates. Thus, in the case of a patient with characteristic symptoms of angina pectoris, the dentist will act promptly and efficiently, administering nitroglycerin to the patient, monitoring at the same time both the patient's blood pressure and his anxiety, through oral sedation (16 ). When there is also an oxygen device in the dentistry office it will be used (the presence of the oxygen device is not obligatory in the dental offices, but it is recommended to exist in the emergency kit, which is mandatory, according to the Romanian legislation in force) (1-6).

In the case of a heart attack that occurred in the dentist's office, the mixed dental team composed of dentist and nurse will call the emergency service 112 , and the patient will be transported quickly by ambulance to a specialized medical unit. But, until the patient is moved to a specialized emergency unit, he or she will be seated and/or horizontal as appropriate, undergo sedation and analgesia, is administered glucose through perfusion and oxygen through the nasal probe, the pulse and blood pressure is monitored (1-6).

If we are talking about a patient with high blood pressure, who presents in a dental office, in case of an acute outbreak of hypertension, the dental treatment will be immediately stopped, the patient being left in a sitting position, the antihypertensive medication he is used to are administered, and when possible, nasal oxygen therapy. The pulse and blood pressure will be constantly monitored. If blood pressure levels continue to be at risk, even after treatment, if symptomatology worsens and complications occur during hemostasis, in this case there is a risk of stroke, cardiac or renal complications, in which case the ambulance is called in emergency (1-6).

At the same time, in atherosclerosis, during dental treatments, the patient with cardiovascular risk may develop vegetative and humoral compensatory mechanisms, which may intensify the heart rhythm and muscular tone (heart failure, coronary insufficiency, heart rhythm disorders etc.). These situations are even more likely in an anxious patient, in the presence of pain and/or due to the anesthetic substance used during therapeutic maneuvers with a dental profile (1-6). 
However, on this occasion, an extremely important aspect must be mentioned, namely that people over the age of 50, at risk of carotid artery atherosclerosis, before starting a usual dental treatment, and even more in the case of more laborious and long-lasting interventions (for example, operations that require the administration of anesthetic substances: endodontic treatments, dento-alveolar surgery such as dental extractions, apical resections, flap operations etc.), should benefit from: a rigorous medical examination (interdisciplinary collaboration between the dentist and the general practitioner) and a conventional radiological examination (orthopantomogram) which, if well performed, could high lighten atheromatous plaques in the carotid artery when they are calcified (7-11).

Thus, we actually tried to familiarize dental practitioners on the therapeutic behavior of patients with atherosclerosis and the risks that arise in it, which are also the purpose of this study, even though we discuss about a preliminary study.

Thus, we actually tried to familiarize dental practitioners on the therapeutic behavior of patients with atherosclerosis and the risks that arise in it, which are also the purpose of this study, even though we discuss about a preliminary study.

\section{MATERIAL AND METHOD}

To conduct this brief study, we composed a questionnaire consisting of 7 points, applied to a number of 87 dentists (simple dentists, resident doctors, specialists and senior in specialties related to dentistry: general dentistry, dental prosthetics, orthodontics and dento-facial orthopedics, pedodontics, endodontics, dento-alveolar surgery, periodontology, oral and maxillo-facial surgery) from Bucharest, Brasov, Rm. Valcea, Alexandria, Pitesti, Constanta and Ploiesti. The subjects ranged in age from 28 to 69 years and had the following gender distribution: 36 of them (representing 41.38\%) were male, while 51 of the subjects (representing

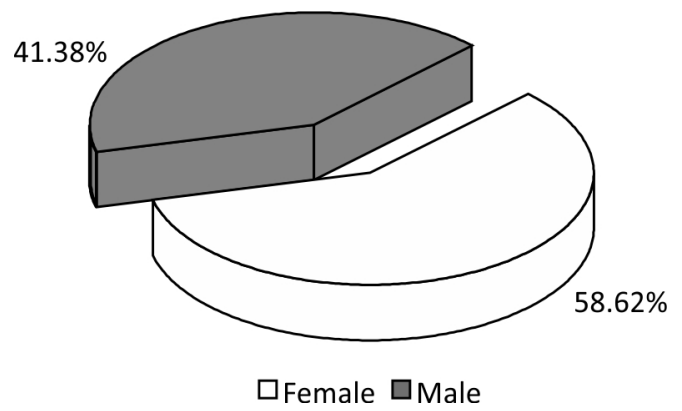

FIGURE 1. Gender distribution of subjects involved in study
$58.62 \%$ ) were female (Fig. 1). The study was conducted between September and November 2018.

In the following part, we will present the questionnaire that we developed.

Questionnaire

1. Changes in the atherosclerosis of the cervical and cerebral arterial system may be associated with risk factors, including: a. High blood pressure; b. Smoking and alcohol consumption; $\boldsymbol{c}$. Obesity, diabetes and dyslipidemia; $\boldsymbol{d}$. Severe dysfunctions of the temporomandibular joint. Correct answers: $a, b, c$.

2. The manifestations of atherosclerosis in the oral cavity consist of: a. Processes of osteoporosis, to which is added the resorption of alveolar processes and changes of the temporomandibular joint; $\boldsymbol{b}$. The epithelium of the oral mucosa thins and keratinizes; $c$. Salivary glands atrophy; d. Salivary secretion decreases; $\boldsymbol{e}$. The interdental relationship change dramatically, with the loss of the vertical dimension of occlusion. Correct answers: $\boldsymbol{a}, \boldsymbol{b}, \boldsymbol{c}, \boldsymbol{d}$.

3. In the case of a patient with symptoms of angina pectoris, the dentist will act as follows: a. He will perform the dental procedures as soon as possible, so that, subsequently, the patient can be sedated; b. will administer to the patient nitroglycerin, while monitoring both the blood pressure and state of anxiety, by oral sedation; $c$. When there is an oxygen device in the dental office, oxygen therapy will also be administered; $\boldsymbol{d}$. will access the 112 emergency service from the first moment. Correct answers: $b, c$.

4. In case of heart attack that occurred in the dentist's office, the mixed dentistry team (dentist-dental nurse) must perform the following actions: a. Will call the emergency service 112, and the patient will be transported quickly with an ambulance to a specialized medical unit; $\boldsymbol{b}$. Until the arrival of the ambulance, the patient will be placed in a sitting and/or horizontal position as appropriate, sedation and analgesia will be administered, perfusion with glucose and oxygen by nasal tube will be administered, and pulse and blood pressure will be monitored; c. Until the arrival of the ambulance, the dental treatments should be completed. Correct answers: $\boldsymbol{a}, \boldsymbol{b}$.

5. If a patient suffering from high blood pressure has an acute outbreak of hypertension during dental treatment, the dentist will perform the following operations: a. Will immediately stop dental treatment, the patient being left in a sitting position, administering the normal antihypertensive medication of the patient; $\boldsymbol{b}$. When possible, nasal oxygen therapy is also administered, with pulse and blood pressure being monitored permanently; c. The den- 
tal treatment is carried on, and in the end the patient will be given an antihypertensive medication; d. Emergency call to the ambulance, when blood pressure levels persist at dangerous levels, even after administration of antihypertensive medication. Correct answers: $a, b, d$.

6. In atherosclerosis, during dental treatments, the patient with cardiovascular risk may develop vegetative and humoral compensatory mechanisms, which may intensify the heart rate and muscular tone (heart failure, coronary insufficiency, heart rhythm disorders, etc.) due to: a. Anxiety; $\boldsymbol{b}$. The presence of pain; $c$. The anesthetic substance used during dental therapeutic maneuvers. Correct answers: $\boldsymbol{a}, \boldsymbol{b}, \boldsymbol{c}$.

7. People over the age of 50, at risk of atherosclerosis in the carotid artery, before starting the usual dental treatment, should benefit from: a. A rigorous medical examination (interdisciplinary collaboration between the dentist and the general practitioner); $\boldsymbol{b}$. A conventional radiological examination (orthopantomogram), which, if well performed, could high lighten atheromatous plaques in the carotid artery when they are calcified; $\boldsymbol{c}$. Psychiatric and/or psychological treatment 3 months before and 3 months after the completion of dental treatment. Correct answers: $\boldsymbol{a}, \boldsymbol{b}$.

\section{RESULTS}

For the first question related to the risk factors that can be associated with changes in the atherosclerosis of the cervical and cerebral arterial system, 77 subjects (representing 88.51\%) answered correctly. The remaining 10 subjects included, besides the correct answers, and the incorrect variant $\mathrm{d}$, the severe dysfunctions of the temporomandibular joint (Fig. 2).

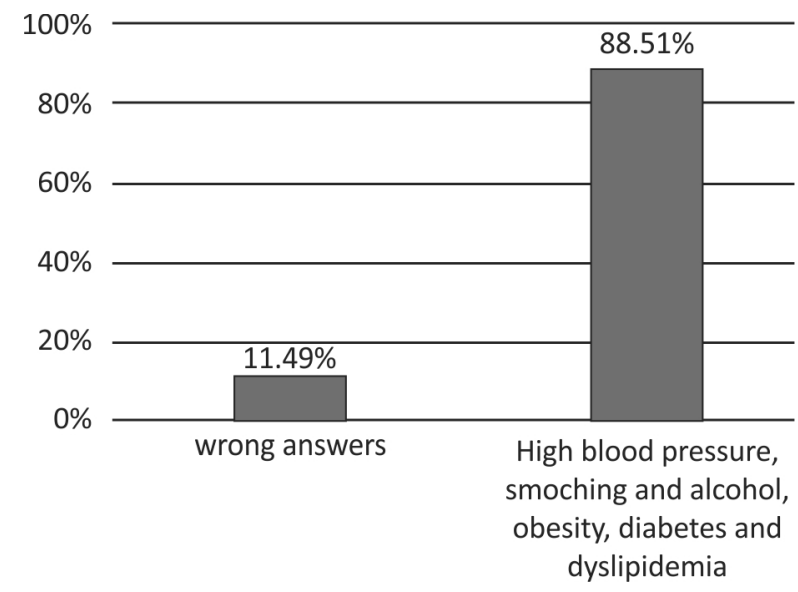

FIGURE 2. Knowledge of risk factors that may be associated with changes in atherosclerosis of the cervical and cerebral arterial system
Regarding the manifestations of atherosclerosis in the oral cavity, only 19 respondents (representing $21.84 \%$ ) answered incorrectly, while the majority (68 respondents representing 78.16\%) answered correctly, namely osteoporosis processes, resorption of alveolar processes, changes of temporomandibular joint, thinning and keratinization of the epithelium of the oral mucosa, atrophy of the salivary glands and decreased salivary secretion (Fig. 3).

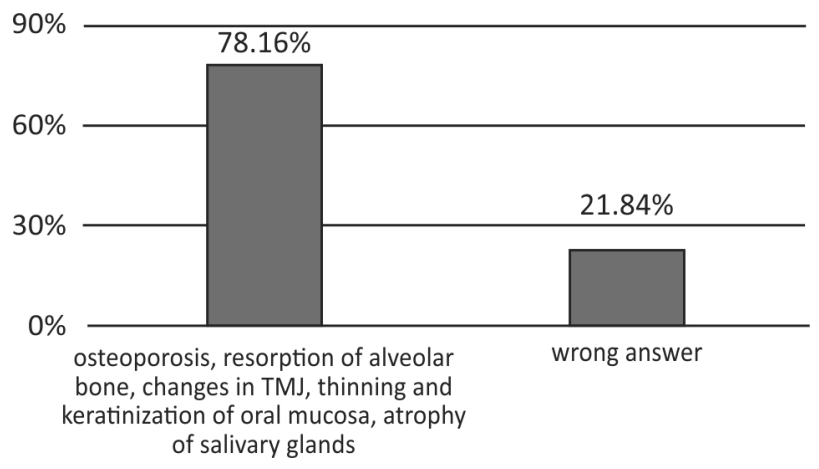

FIGURE 3. Understanding the manifestations of atherosclerosis at the level of the oral cavity

For the third question regarding how to act in the case of a patient with angina pectoris symptoms, 80 of the practitioners (representing 91.95\%) answered correctly (administration of nitroglycerin, monitoring vital signs and administration of oxygen therapy where possible). A small number of practitioners ( 7 representing $8.05 \%$ ) included besides the correct answer variants and the one that involves calling the emergency service from the first moment. We can correlate these responses with the age of the patients, those with more experience in practice approaching such a situation in a more temperate manner and the younger ones, with less experience, exaggerate using all the tools available for the given situation (Fig. 4).

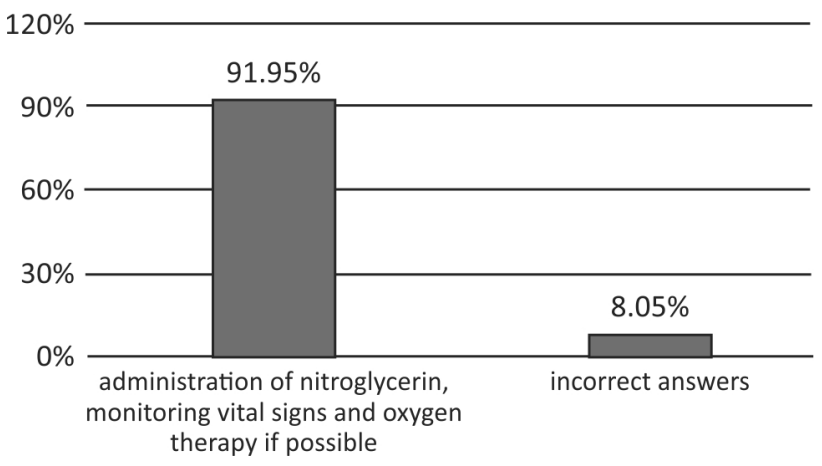

FIGURE 4. Attitude towards a patient with angina pectoris symptoms

It should be noted that all doctors included in the study answered correctly about the attitude towards 
a patient with heart attack that occurred in the dental office.

The attitude of the practitioners towards the situation in which a patient undergoing dental treatment has a outbreak of hypertension was the following: 73 practitioners (representing 83.91\%) answered correctly (discontinuation of treatment, administration of antihypertensive medication, oxygen therapy, emergency ambulance call), while only 14 practitioners (representing 16.09\%) excluded the ambulance call response (Fig. 5).

Concerning the factors that may develop vegetative and humoral compensatory mechanisms during dental treatments in patients with cardiovascular risk, all respondents to this study answered correctly (anxiety, pain and anesthetic substances used).
For the last question related to the examinations recommended for patients over 50 years old with risk of carotid artery atherosclerosis before the start of dental treatments, most of the doctors included in the study ( 83 - representing $95.40 \%$ ) answered correctly (medical examination and conventional radiologic examination well done) while only 4 doctors (representing 4.60\%) responded incorrectly all variants (Fig. 6).

\section{DISCUSSIONS}

Dentistry does not exist as a completely isolated science, it is strongly linked to other medical branches. Thus, any deficiency at this level finds its effect in the body and also the affections of the other systems have an effect at the level of the oral cavity.

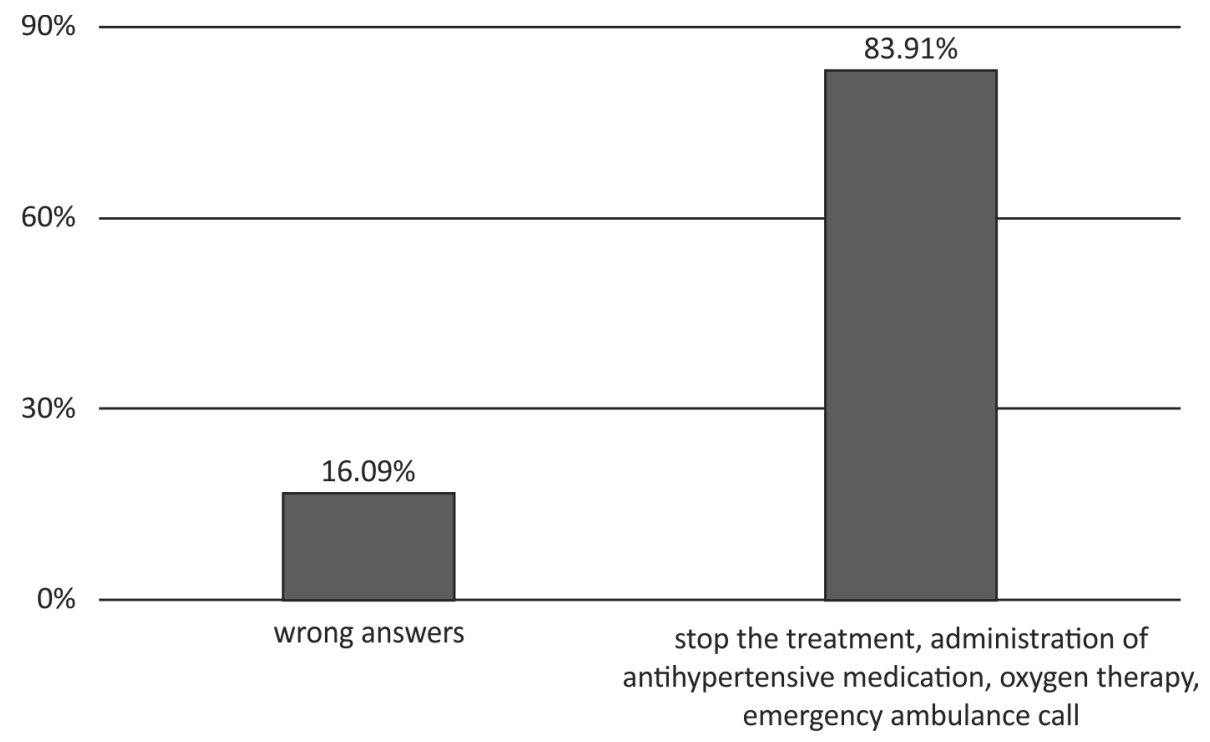

FIGURE 5. Procedures to be followed in case of a hypertensive outbreak

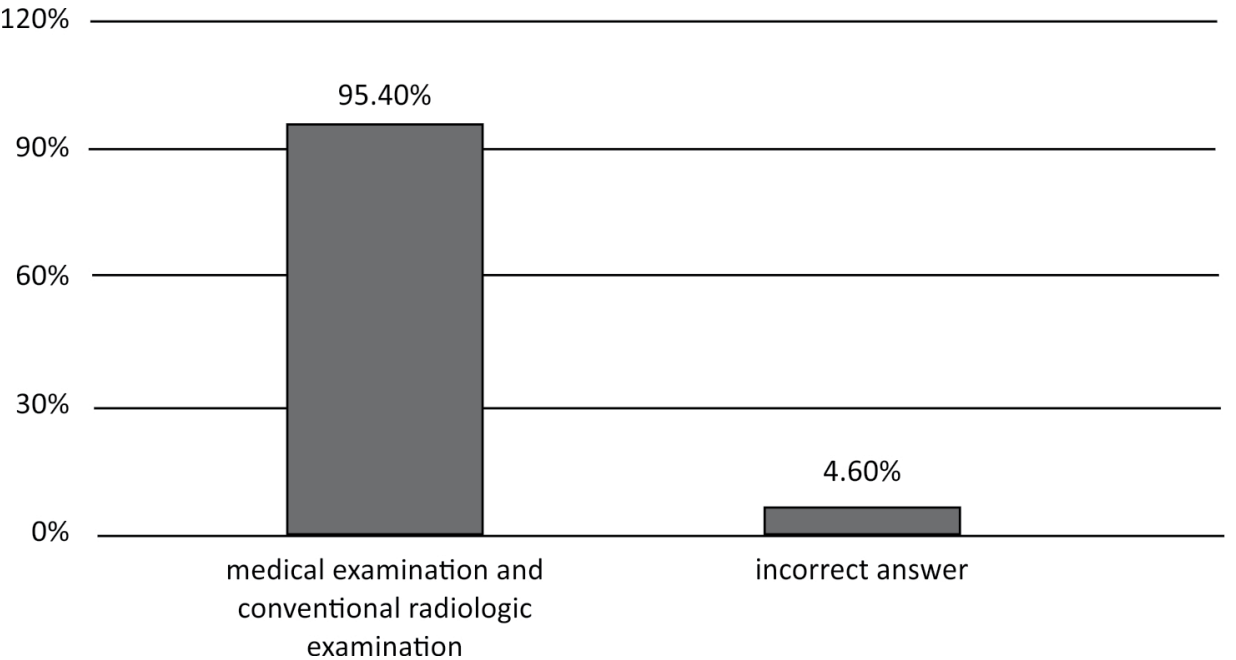

FIGURE 6. Recommended examinations for patients at risk of atherosclerosis in the carotid artery 
Atherosclerosis is a disease in which plaque builds up inside arteries. In time, plaque hardens and narrows arteries, and this limits the flow of oxygen-rich blood to organs and other parts of the body. Atherosclerosis can lead to serious problems, including heart attack, stroke, or even death (12).

"Four basic pathogenic mechanisms have been proposed that involve oral inflammations in the pathogenesis of atherosclerosis: low-level bacteremia, systemic inflammation induced by inflammatory mediators, autoimmunity to host proteins which results from the host immune response to specific components of oral pathogens, and pro-atherogenic effects resulting from specific bacterial toxins that are produced by oral pathogenic bacteria" (13).

The connection between atherosclerosis and oral cavity is almost related to effects of oral infection in producing mechanism of atherosclerosis. Dankevych-Kharchyshyn et al., in a 2019 literature review, concluded that "there is a clear and directly proportional relationship between periodontal diseases and atherosclerosis" (14). Regarding the reverse connection, between atherosclerosis and oral cavity, there are a few data mainly because the attention of medical stuff is focused on general symptoms of atherosclerosis especially on symptoms that precede heart attack.

Therefore, the dentist should know atherosclerosis symptoms that may precede a heart attack, and also other signs that may appear on regular medical exams connected to dental treatments as x-rays. Guimarães Henriques et al., in a study from 2011, highlighted the aspect of carotid artery atheromas on panoramic radiography (16). That's why, even if is not an exam of choice, dental practitioner can identify on a panoramic radiography the carotid artery atheromas and subsequent to guide the patient for a specific medical evaluation for diagnose and treatment.

Being a risk group, the treatment and attitude of dentists towards such patients must follow a well-defined path to prevent any exacerbation of the underlying condition and reduce the number of risk factors in the oral cavity. Thus, numerous studies and practice guides have been outlined by the profile associations. Singh et al., in a literature review, draw some dental management protocols for cardiovascular compromised patients with direct referral to anesthetic solutions to be used and also oxygen therapy protocol in case of a stroke (16). In 2002, Rose et al. described dental management for patients with cardiovascular disease depending on affected level and medication (17). In the same manner, Jowett et al. concluded in their study that dental team should approach the patients with cardiovascular risk with attention before and during the treatment. "In case of developing significant symptoms, all treatments should be stop, and the practitioner should be prepared to initiate emergency care" (18).

The practice guide, as the studies show, is absolutely necessary and must be known by all dentists given the fact that the number of patients with such conditions is constantly increasing. Our study, like many other studies, tried to make dental practitioners aware of this problem, especially since most practitioners perform treatments in private clinics, separate from hospitals, from the disinterest of the health system on this medical branch.

\section{CONCLUSIONS}

All dentists have the obligation to know the pathology of patients with cardiovascular risk after atherosclerosis, given the high frequency of patients suffering from atherosclerotic lesions.

Knowledge by dentists of general prophylactic measures of atherosclerosis, including those in the oral and maxillofacial area, including the morphofunctional integrity of the dento-maxillary system, plays an essential role in maintaining the quality of life of each individual.

It is mandatory for each dental office in Romania to have an emergency kit, according to the legislation in force.

There is an indisputable link between periodontitis and atherosclerosis. Basically, this hypothesis about the role of inflammation contributes to establishing a more complete etiological diagnosis of atherosclerosis, allowing the improvement of its treatment.

Dental treatments in patients at cardiovascular risk following atherosclerosis must take into account vascular risk factors, the removal of the periodontal inflammatory factor and, only subsequently treating the dental and periodontal lesions.

Endodontic and dento-alveolar surgery interventions in this type of patients must be resolved in collaboration with the cardiologist and/or neurologist as appropriate, to avoid unpleasant accidents or the occurrence of life-threatening complications for the patient.

\section{Acknowledgement}

In this article, all the authors have equal contribution with the first author. 


\section{REFERENCES}

1. Bodnar DC și colab. Managementul dentar al pacientului cu probleme medicale complexe. Editura Ars Docendi, București, 2012.

2. Voroneanu M, Bucur A, lordache N, Bălan H. Urgențe medico-chirurgicale în cabinetul de medicină dentară. Editura Medicală, București, 2016.

3. Bodnar DC. Modificări dento-parodontale și maxilare în afecțiunile nevraxiale. Teză de doctorat, U.M.F. „Carol Davila”, București, 2005.

4. Burlibașa M, Bodnar DC. Aspecte teoretice și practice privind corelația dintre diferitele afecțiuni generale și managementul general al pacientului. În: Progrese în medicina dentară, vol. VI. Coordonatori. Dumitrache AM, Burlibașa M, Sfeatcu RI, Bodnar T, Editura Ars Docendi, București, 2012, pp. 45-54.

5. Sfeatcu $R$, Burlibaşa $M$, Tofan $C$ et al. Calitatea vieţii, un concept multidisciplinar. Sibiul Medical. 2007;18(1):18-20.

6. Little WJ, Falace AD, Miller SC, Rhodus LN. Dental management of the medically compromised patient. Seventh Edition - Mosby Elsevier, 2008.

7. Eftimie-Totu E, Cristache CM, Isildak S et al. Preliminary studies on citotoxicity and genotoxicity assesment of the PMMA - TiO2 nanocompozites for stereolithographic complete dentures manufacturing. Revista de Chimie. 2018;69(5):1160-1165.

8. Bodnar DC, Burlibașa L, Vârlan C et al. Mercury, biocompatibility and its impact on environment. Metalurgia International. 2009;14:95-100.

9. Burlibașa M, Tănase G, Muntianu L et al. Quality of life, a multidisciplinary concept with economic and social impacts in medical practice. Metalurgia International. 2010;15(4):88-90.
10. Mocuța D, Popovici IA, Burlibașa $L$ et al. Impact of the living conditions on population health. Metalurgia International. 2009;14:1719.

11. Ionescu CA, Popovici LR, Mocuța $D$ et al. The quality of human life from the perspective of sustainable development. Metalurgia International. 2009;14:41-43.

12. National Heart, Lung, and Blood Institute. Atherosclerosis. Health topics -atherosclerosis. [Online] [Cited: 15.03.2020.] https://www. nhlbi.nih.gov/health-topics/atherosclerosis.

13. Aarabi G, Heydecke G, Seedorf U. Roles of Oral Infections in the Pathomechanism of Atherosclerosis. Int J Mol Sci. 2018;19(7):1978.

14. Dankevych-Kharchyshyn IS, Vynogradova OM, Malko NV et al. Periodontal diseases and atherosclerosis (literature review). Wiad Lek. 2019;72(3):462-465.

15. Guimarães Henriques JC, Kreich EM, Helena Baldani M, Luciano M, Cezar de Melo Castilho J, Cesar de Moraes L. Panoramic radiography in the diagnosis of carotid artery atheromas and the associated risk factors. Open Dent J. 2011;5:79-83.

16. Singh S, Gupta K, Garg KN et al. Dental Management of the Cardiovascular Compromised Patient: A Clinical Approach. J Young Pharmacists. 2017;9(4):453-456.

17. Rose LF, Mealey B, Minsk L, Cohen DW. Oral care for patients with cardiovascular disease and stroke. J Am Dent Assoc. 2002;133(Suppl):37S-44S.

18. Jowett NI, Cabot LB. Patients with cardiac disease: Considerations for the dental practitioner. Br Dent J. 2000;189(6):297-302.

Conflict of interest: none declared Financial support: none declared 